\title{
THE COMPLEXITIES OF MODERN MEDICINE
}

\section{Tan SY}

Nephrology \& Renal Transplant Services, Prince Court Medical Centre., Kuala Lumpur, Malaysia.

The current issue of JUMMEC touches on many diverse topics and in many ways reflects the evolution of modern medicine from the practice of acupuncture to epidemics facilitated by modern travel to the subject of ethics including controversies surrounding financial incentives given in promoting organ donation.

The review on neonatal cholestasis and biliary atresia (1) illustrate the challenges of clinical practice in a developing country. The need for early diagnosis is obvious and cannot be over emphasized. Whilst early diagnosis is crucial to effective treatment, such treatment may not be routinely available in a developing country. All too frequently, the development of a country is often judged by is physical infrastructure and services such as education and healthcare are commonly lagging behind. Equipment which are frequently expensive and found only in developed countries may be available for early diagnosis but this is effectively useful for curative treatment by transplantation only if health services such as liver transplantation are routinely available. This is currently being addressed and Malaysia is fortunate in having a national liver transplant programme that is evolving and expanding to meet the increasing demand for liver transplant services.

Healthcare services worldwide have been increasingly challenged by epidemics caused by new viruses, the transmission of which are increased by easy access to modern mass travel and migration from rural areas to increasingly crowded cities. In the West several years ago, there was an outbreak of mad cow disease otherwise known as bovine spongiform encephalopathy which is spread by consumption of contaminated meat (2). Cattle which are normally herbivores were fed food contaminated with viruses, usually found in infected brain and neural tissue, leading to infection in cattle meat which then spread to humans. More recently in Malaysia, an outbreak of fatal infection mainly involving individuals especially farmers who came into contact with contaminated pigs (3) led to massive culling of infected animals and reorganization of health services to deal with this public health issue. This was accompanied by bird flu and more recently H1N1 infection (4). The impact on the economy, air travel, social behaviour and health services was obvious. A new cause of ARDS was indentified in another review article in this issue relating to H1N1 infection (5). In common with the article on biliary atresia, early diagnosis and aggressive intervention was crucial for improved survival with the potential application of ECMO in patients affected by ARDS discussed. Such therapy however, are not routinely available in many countries and this deficiency together with the increasing challenge of H1N1 and related infections causing ARDS, meant that realignment of healthcare budget to meet such challenges may be necessary.

The ancient practice of acupuncture continues to demonstrate it's relevance despite the advances of modern medicine. The wide range of diseases and symptoms which may benefit from acupuncture are reinforced in the article on practice of acupuncture in Saudi Arabia (6) which also illustrate the most commonly held public perception on the role of acupuncture in providing pain relief. The effectiveness of a clinical procedure therefore, need not be modern or expensive to be effective as clearly illustrated by the practice of acupuncture.

The papers on ethics (7) and enhancing organ donor pool through financial incentives (8) are clearly related and reflect the increasingly important role of an effective ethics committee in modern medicine especially with regards to clinical research and practice of organ donation. The role of organ transplantation in saving lives is undisputed and it is also clear that the disparity between demand and the number of potential donors is increasing particularly with the lack

\footnotetext{
Correspondence:

Tan Si Yen

Head of Nephrology \& Renal Transplant Services

Prince Court Medical Centre

50450 Kuala Lumpur

Email: siyen.tan@princecourt.com
} 
of cadaver organ donors partly contributed to by the effectiveness of modern transportation and road safety laws in reducing accidents. The controversies in the use of alternative sources of organ donors that patients and doctors worldwide have turned to in their increasingly desperate effort to have life saving organ transplants remain a challenge. Organ transplant programs the world over are now looking more and more at unrelated donors and most countries have attempted to regulate the potential abuse of commercially driven unrelated transplants by the use of ethics committees and guidelines to protect such donors. As a result, transplant programs in most developed countries are seeing an increasingly large proportion of transplants performed where the organs are from an unrelated donor. This practice is currently not encouraged in Malaysia although the issue is being addressed by the setting up of an Unrelated Transplant Action Committee (UTAC) by the Ministry of Health where potential unrelated donors may be considered on a case by case basis. The ethics and the controversies surrounding unrelated transplants remain difficult and probably impossible to resolve. Those against unrelated transplant programs are concerned about the potential abuse of poor donors who may not fully understand the procedures involved. On the other end, supporters of the program are reassured by the relatively safe procedures of kidney donation in good hands and the supervision provided by an effective ethics committee. In addition, arguments are often raised that while it is usually the financially disadvantaged individuals who are donors, such individuals are also more likely to serve in dangerous occupations where the potential danger to their health and safety is more relevant than being an organ donor.

In summary, the diversity of articles in the current issue of JUMMEC reflects the evolution of modern medicine without compromising traditional effective therapy such as acupuncture. New diseases which may be easily spread by modern mass communication are now posing increasing challenges to our healthcare systems. The ethics and controversy surrounding clinical research and organ transplantation are still being addressed and Hippocrates in his teaching may have anticipated this problem by his own demands in his oath (9) that doctors must "first do no harm".

\section{References}

1. 1. Lee WS. Neonatal cholestasis and biliary atresia: perspective from Malaysia. JUMMEC 2010; 13(2): 72-79.

2. Harman JL, Silva CJ. Bovine spongiform encephalopathy. J Am Vet Med Assoc 2009 Jan 1; 234(1): 59-72.

3. Chua KB, Goh KJ, Wong KT et al. Fatal encephalitis due to Nipah virus among pig-farmers in Malaysia. Lancet 1999; 354: 1257-1259.

4. Lee CK. Influenza A (H1N1) 2009 pandemic virus: learning from the first wave, preparing for the second. Med J Malaysia 2010 Mar; 65(1): 1-2.

5. Ismail AH, Marzida M, Kumar NM, Ong GSY. H1N1 Induced ARDS: ECMO as Rescue Therapy in Patients with Failed Mechanical Ventilation JUMMEC 2010; 13 (2): 80-87.

6. AlRukhban MO. The Practice of Acupuncture in Saudi Arabia. JUMMEC 2010; 13 (2): 93-101.

7. AlKaabba A. Introducing Clinical Ethics Consultation Service to King Fahad Medical City in Riyadh Kingdom of Saudi Arabia. JUMMEC 2010; 13 (2): 88-92.

8. Tumin M, Ndoma I. Enhancing the Donor Pool. JUMMEC 2010; 13 (2): 102-106.

9. Eliot CW. Harvard Classics: Volume 38. NY: Collier \& Son; 1910. 